\title{
Strategies to Optimize Order within Planar Arrays of Myelin Basic Protein for Electron Crystallography
}

\author{
C. M. Hill, I. R. Bates", C. E. Antler , G. F. White , F. R. Hallett ${ }^{* *}$ and G. Harauz \\ Departments of "Molecular Biology \& Genetics, and ${ }^{* *}$ Physics, an d Biophysics Interdepartmental \\ Group, University of Guelph, 50 Stone Road East, Guelph, Ontario, Canada, N1G 2W1.
}

Knowledge of the tertiary structure of myelin basic protein (MBP), and its organisation within normal compacted and disrupted myelin multilayers, is essential to understanding the organisation of the myelin sheath and the mechanisms of development of autoimmunity in multiple sclerosis [4]. Previously, we have employed a functionalised lipid monolayer as a substrate to produce planar arrays of hexahistidine -tagged recombinant murine MBP [1,3]. Optimal crystallization conditions were determined using a stepwise approach to sample incubation time, temperature, monolayer composition, buffer composition (including incorporation of divalent cations and free imidazole) and $\mathrm{pH}$. The choice of diluting lipid incorporated into the substrate monolayer proved to be the most important variable, and phosphoinositides in particular were shown to produce planar arrays of anchored MBP [3].

MBP contains lit tle secondary structure in aqueous solution and can be classified as an intrinsically disordered protein. Thus, MBP has been extremely refractory to crystallization attempts [5]. Here, we have attempted to improve the crystallinity of our two -dimensional crystals by attenuating the flexibility of the protein. We have employed lipids as biological chaperones and osmolytes as chemical chaperones in an attempt to maximize the proportion of regular secondary structure within MBP. MBP deiminated in vitro to mimic the natural deiminated form found in individuals suffering from multiple sclerosis has been shown to exhibit greater order in crystallization experiments [3]. A further quasi-deiminated recombinant form of MBP containing six Arg/Lys-> Gln substitutions has been generated to produce a more homogeneous, reproducible population of this less cationic variant [2]. Deletion mutants have also been constructed to remove both termini, regions predicted to be disordered by neural network analysis. Finally, dyn amic light scattering and circular dichroism have been employed to monitor the aggregation and conformation of MBP populations used for crystallization.

Inclusion of the naturally -occuring osmolyte trimethylamine $N$-oxide (Fig. 1) resulted in noticeable improvement over duplicate experiments omitting osmolytes (Fig. 2). The Arg ->Gln mutant seemed to have a predisposition towards layered growth (Fig. 3).

References and Acknowledgements

[1] I.R. Bates et al., Prot. Expr. Purif. 20 (2000) 285.

[2] I.R. Bates et al., Prot. Expr. Purif. (2002) submitted.

[3] N. Ishiyama et al., J. Struct. Biol. 136 (2001) in press.

[4] M.A. Moscarello, in Cell Biology and Pathology of Myelin: Evolving Biological Concepts and Therapeutic Approaches, pp. 13-25, Plenum Press, New York, 1997.

[5] J. Sedzik and D.A. Kirschner, Neurochem. Res. 17 (1992) 157.

[6] This work was supported by the Canadian Institutes for Health Research, the Multiple Sclerosis Society of Canada, and the Natural Sciences and Engineering Research Council of Canada. 

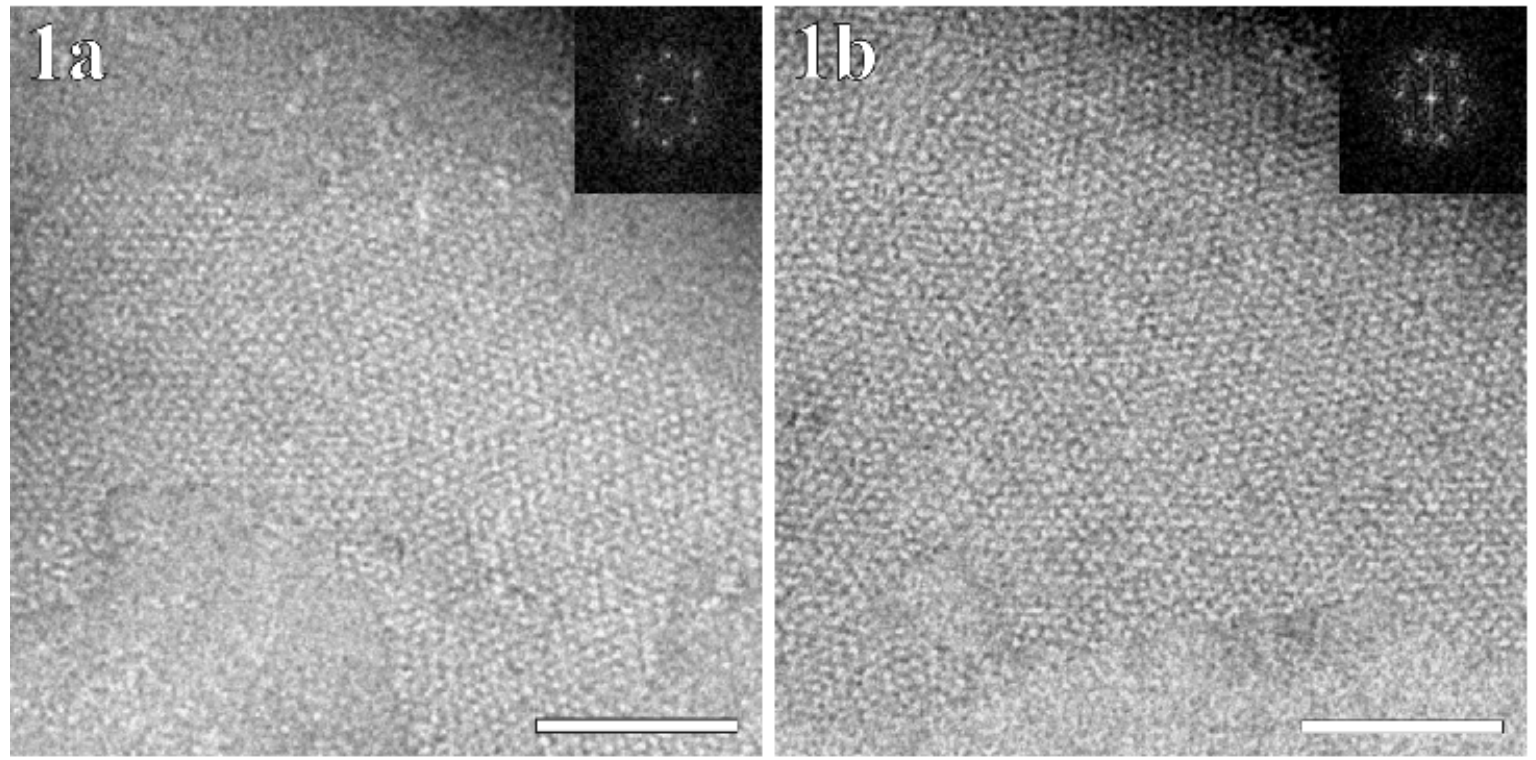

Fig. 1. (a, b) Planar crystalline arrays of recombinant murine MBP on a monolayer substrate consisting of nickel-chelating lipid and phosphatidylinositol 4-phosphate in the presence of the osmolytes trimethylamine $N$-oxide and L-proline. Scale bars represent $100 \mathrm{~nm}$.

Insets: Fourier transforms of well-ordered regions within the arrays.
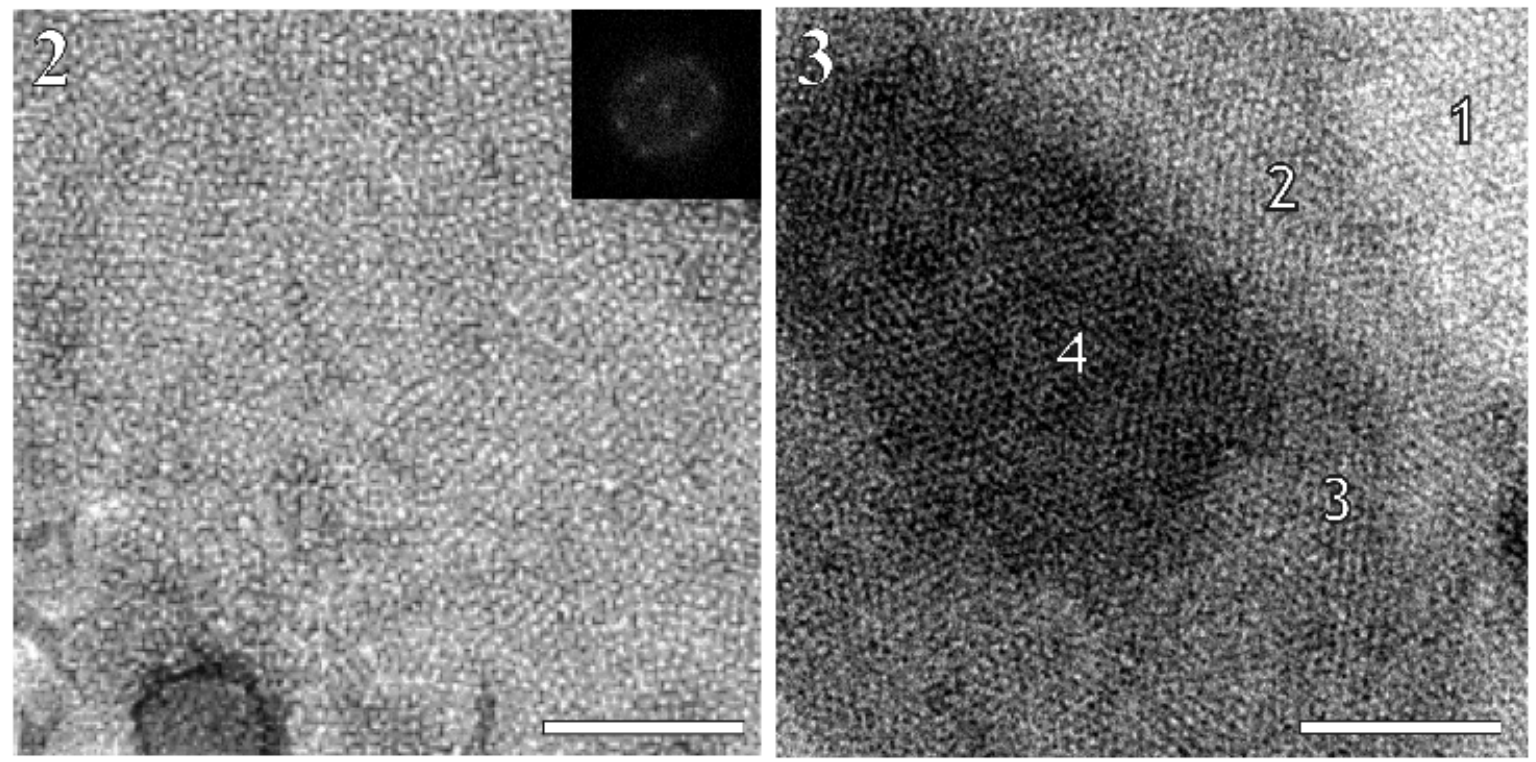

Fig. 2. Planar crystalline arrays of recombinant murine myelin basic protein on a monolayer substrate consisting of an equal proportion of nickel-chelating lipid and phosphatidylinositol 4-phosphate in the absence of any osmolytes. Scale bar represents $100 \mathrm{~nm}$. Inset: Fourier transform of best-ordered region.

Fig. 3. Layered planar arrangement of Arg->Lys mutated MBP on a monolayer substrate consisting of nickel-chelating lipid and phosphatidylinositol 4-phosphate in the presence of trimethylamine $N$-oxide and L-proline. The numbers of layers in each density region are labelled. Scale bar represents $100 \mathrm{~nm}$. 